Supporting Information for

\title{
Inhibitive Chain Transfer to Ligand in the ATRP of $n$-Butyl Acrylate
}

\author{
Rahul Sharma, Ayush Goyal, James M. Caruthers, and You-Yeon Won* \\ School of Chemical Engineering, Purdue University, West Lafayette, Indiana 47907
}

*yywon@ecn.purdue.edu 
Section 1: Kinetic balance equations for modeling the ATRP of nBA using the conventional ATRP mechanism.

$$
\begin{aligned}
& \frac{\mathrm{d}[\mathrm{IBr}]}{\mathrm{dt}}=\mathrm{k}_{\mathrm{d}}[\mathrm{I} \bullet]\left[\mathrm{CuBr}_{2}\right]-\mathrm{k}_{\mathrm{a}}[\mathrm{IBr}][\mathrm{CuBr}] \\
& \frac{\mathrm{d}[\mathrm{I} \bullet}{\mathrm{dt}}=-\mathrm{k}_{\mathrm{d}}[\mathrm{I} \bullet]\left[\mathrm{CuBr}_{2}\right]+\mathrm{k}_{\mathrm{a}}[\mathrm{IBr}][\mathrm{CuBr}]-\mathrm{k}_{\mathrm{p}}[\mathrm{I} \bullet][\mathrm{M}]-\frac{1}{2}\left(\mathrm{k}_{\mathrm{tc}, \mathrm{I}}+\mathrm{k}_{\mathrm{tc}}\right)[\mathrm{I} \bullet]\left[\lambda_{0}\right]-\mathrm{k}_{\mathrm{tc}, \mathrm{I}}[\mathrm{I} \bullet]^{2} \\
& \frac{\mathrm{d}\left[\lambda_{0}\right]}{\mathrm{dt}}=-\mathrm{k}_{\mathrm{d}}\left[\lambda_{0}\right]\left[\mathrm{CuBr}_{2}\right]+\mathrm{k}_{\mathrm{a}}\left[\mu_{0}\right][\mathrm{CuBr}]+\mathrm{k}_{\mathrm{p}}[\mathrm{I} \bullet][\mathrm{M}]-\frac{1}{2}\left(\mathrm{k}_{\mathrm{tc}, \mathrm{I}}+\mathrm{k}_{\mathrm{tc}}\right)[\mathrm{I} \bullet]\left[\lambda_{0}\right]-\mathrm{k}_{\mathrm{tc}}\left[\lambda_{0}\right]^{2} \\
& \frac{\mathrm{d}\left[\lambda_{1}\right]}{\mathrm{dt}}=-\mathrm{k}_{\mathrm{d}}\left[\lambda_{1}\right]\left[\mathrm{CuBr}_{2}\right]+\mathrm{k}_{\mathrm{a}}\left[\mu_{1}\right][\mathrm{CuBr}]+\mathrm{k}_{\mathrm{p}}[\mathrm{I} \bullet][\mathrm{M}]-\frac{1}{2}\left(\mathrm{k}_{\mathrm{tc}, \mathrm{I}}+\mathrm{k}_{\mathrm{tc}}\right)[\mathrm{I} \bullet]\left[\lambda_{1}\right]-\mathrm{k}_{\mathrm{tc}}\left[\lambda_{0}\right]\left[\lambda_{1}\right]+ \\
& \mathrm{k}_{\mathrm{p}}\left[\lambda_{0}\right][\mathrm{M}] \\
& \frac{\mathrm{d}\left[\lambda_{2}\right]}{\mathrm{dt}}=-\mathrm{k}_{\mathrm{d}}\left[\lambda_{2}\right]\left[\mathrm{CuBr}_{2}\right]+\mathrm{k}_{\mathrm{a}}\left[\mu_{2}\right][\mathrm{CuBr}]+\mathrm{k}_{\mathrm{p}}[\mathrm{I} \bullet][\mathrm{M}]-\frac{1}{2}\left(\mathrm{k}_{\mathrm{tc}, \mathrm{I}}+\mathrm{k}_{\mathrm{tc}}\right)[\mathrm{I} \bullet]\left[\lambda_{2}\right]-\mathrm{k}_{\mathrm{tc}}\left[\lambda_{0}\right]\left[\lambda_{2}\right]+ \\
& \mathrm{k}_{\mathrm{p}}\left[\lambda_{0}\right][\mathrm{M}]+2 \mathrm{k}_{\mathrm{p}}\left[\lambda_{1}\right][\mathrm{M}] \\
& \frac{\mathrm{d}\left[\mu_{0}\right]}{\mathrm{dt}}=\mathrm{k}_{\mathrm{d}}\left[\lambda_{0}\right]\left[\mathrm{CuBr}_{2}\right]-\mathrm{k}_{\mathrm{a}}\left[\mu_{0}\right][\mathrm{CuBr}] \\
& \frac{\mathrm{d}\left[\mu_{1}\right]}{\mathrm{dt}}=\mathrm{k}_{\mathrm{d}}\left[\lambda_{1}\right]\left[\mathrm{CuBr}_{2}\right]-\mathrm{k}_{\mathrm{a}}\left[\mu_{1}\right][\mathrm{CuBr}] \\
& \frac{\mathrm{d}\left[\mu_{2}\right]}{\mathrm{dt}}=\mathrm{k}_{\mathrm{d}}\left[\lambda_{2}\right]\left[\mathrm{CuBr}_{2}\right]-\mathrm{k}_{\mathrm{a}}\left[\mu_{2}\right][\mathrm{CuBr}] \\
& \frac{\mathrm{d}\left[\chi_{0}\right]}{\mathrm{dt}}=\frac{1}{2}\left(\mathrm{k}_{\mathrm{tc}, \mathrm{I}}+\mathrm{k}_{\mathrm{tc}}\right)[\mathrm{I} \bullet]\left[\lambda_{0}\right]+\frac{\mathrm{k}_{\mathrm{tc}}}{2}\left[\lambda_{0}\right]^{2} \\
& \frac{\mathrm{d}\left[\chi_{1}\right]}{\mathrm{dt}}=\frac{1}{2}\left(\mathrm{k}_{\mathrm{tc}, \mathrm{I}}+\mathrm{k}_{\mathrm{tc}}\right)[\mathrm{I} \bullet]\left[\lambda_{1}\right]+\mathrm{k}_{\mathrm{tc}}\left[\lambda_{0}\right]\left[\lambda_{1}\right] \\
& \frac{\mathrm{d}\left[\chi_{2}\right]}{\mathrm{dt}}=\frac{1}{2}\left(\mathrm{k}_{\mathrm{tc}, \mathrm{I}}+\mathrm{k}_{\mathrm{tc}}\right)[\mathrm{I} \bullet]\left[\lambda_{2}\right]+\mathrm{k}_{\mathrm{tc}}\left(\left[\lambda_{0}\right]\left[\lambda_{2}\right]+\left[\lambda_{1}\right]^{2}\right) \\
& \frac{\mathrm{d}[\mathrm{CuBr}]}{\mathrm{dt}}=\mathrm{k}_{\mathrm{d}}\left(\left[\lambda_{0}\right]+[\mathrm{I} \bullet]\right)\left[\mathrm{CuBr}_{2}\right]-\mathrm{k}_{\mathrm{a}}\left(\left[\mu_{0}\right]+[\mathrm{IBr}]\right)[\mathrm{CuBr}] \\
& \frac{\mathrm{d}[\mathrm{M}]}{\mathrm{dt}}=-\mathrm{k}_{\mathrm{p}}\left[\lambda_{0}\right][\mathrm{M}]-\mathrm{k}_{\mathrm{p}}[\mathrm{I} \bullet][\mathrm{M}] \\
& {[\mathrm{CuBr}]+\left[\mathrm{CuBr}_{2}\right]=[\mathrm{CuBr}]_{0}} \\
& {\left[\lambda_{\mathrm{i}}\right]=\sum_{\mathrm{n}=1}^{\infty} \mathrm{n}^{\mathrm{i}}\left[\mathrm{R}_{\mathrm{n}} \bullet\right] \quad \mathrm{i}=1,2,3} \\
& {\left[\mu_{\mathrm{i}}\right]=\sum_{\mathrm{n}=1}^{\infty} \mathrm{n}^{\mathrm{i}}\left[\mathrm{R}_{\mathrm{n}} \mathrm{Br}\right] \quad \mathrm{i}=1,2,3} \\
& {\left[\chi_{\mathrm{i}}\right]=\sum_{\mathrm{n}=1}^{\infty} \mathrm{n}^{\mathrm{i}}\left[\mathrm{P}_{\mathrm{n}}\right] \quad \mathrm{i}=1,2,3}
\end{aligned}
$$


$\mathrm{k}_{\mathrm{tc}}=\mathrm{k}_{\mathrm{tc} 0} \mathrm{DP}^{-(0.065+0.016 \mathrm{DP})}$

$\mathrm{DP}=113+\frac{[\mathrm{M}]_{0}}{[\mathrm{I}]_{0}} \mathrm{x}$

$\mathrm{x}=1-\frac{[\mathrm{M}]}{[\mathrm{M}]_{0}}$

$\mathrm{k}_{\mathrm{tc}, \mathrm{I}}=\mathrm{k}_{\mathrm{tc}}(\mathrm{x}=0)$

Section 2: Kinetic balance equations for modeling the ATRP of nBA on the basis of the mechanism that accounts for the chain transfer to PMDETA.

$$
\begin{aligned}
& \frac{\mathrm{d}[\mathrm{IBr}]}{\mathrm{dt}}=\mathrm{k}_{\mathrm{d}}[\mathrm{I} \bullet]\left[\mathrm{CuBr}_{2}\right]-\mathrm{k}_{\mathrm{a}}[\mathrm{IBr}][\mathrm{CuBr}] \\
& \frac{\mathrm{d}[\mathrm{I} \bullet}{\mathrm{dt}}=-\mathrm{k}_{\mathrm{d}}[\mathrm{I} \bullet]\left[\mathrm{CuBr}_{2}\right]+\mathrm{k}_{\mathrm{a}}[\mathrm{IBr}][\mathrm{CuBr}]-\mathrm{k}_{\mathrm{p}}[\mathrm{I} \bullet][\mathrm{M}]-\mathrm{k}_{\mathrm{tr}}[\mathrm{I} \bullet]\left([\mathrm{L}]+\left[\mathrm{L}^{=}\right]\right)-\mathrm{k}_{\mathrm{tl}}[\mathrm{I} \bullet] \\
& \left([\mathrm{L} \bullet]+\left[\mathrm{L}^{=} \bullet\right]\right)-\frac{1}{2}\left(\mathrm{k}_{\mathrm{tc}, \mathrm{I}}+\mathrm{k}_{\mathrm{tc}}\right)[\mathrm{I} \bullet]\left[\lambda_{0}\right]-\mathrm{k}_{\mathrm{tc}, \mathrm{I}}[\mathrm{I} \bullet]^{2} \\
& \frac{\mathrm{d}\left[\lambda_{0}\right]}{\mathrm{dt}}=-\mathrm{k}_{\mathrm{d}}\left[\lambda_{0}\right]\left[\mathrm{CuBr}_{2}\right]+\mathrm{k}_{\mathrm{a}}\left[\mu_{0}\right][\mathrm{CuBr}]+\mathrm{k}_{\mathrm{p}}[\mathrm{I} \bullet][\mathrm{M}]-\mathrm{k}_{\mathrm{tr}}\left[\lambda_{0}\right]\left([\mathrm{L}]+\left[\mathrm{L}^{=}\right]\right)-\mathrm{k}_{\mathrm{tl}}\left[\lambda_{0}\right] \\
& \left([\mathrm{L} \bullet]+\left[\mathrm{L}^{=} \bullet\right]\right)-\frac{1}{2}\left(\mathrm{k}_{\mathrm{tc}, \mathrm{I}}+\mathrm{k}_{\mathrm{tc}}\right)[\mathrm{I} \bullet]\left[\lambda_{0}\right]-\mathrm{k}_{\mathrm{tc}}\left[\lambda_{0}\right]^{2} \\
& \frac{\mathrm{d}\left[\lambda_{1}\right]}{\mathrm{dt}}=-\mathrm{k}_{\mathrm{d}}\left[\lambda_{1}\right]\left[\mathrm{CuBr}_{2}\right]+\mathrm{k}_{\mathrm{a}}\left[\mu_{1}\right][\mathrm{CuBr}]+\mathrm{k}_{\mathrm{p}}[\mathrm{I} \bullet][\mathrm{M}]-\mathrm{k}_{\mathrm{tr}}\left[\lambda_{1}\right]\left([\mathrm{L}]+\left[\mathrm{L}^{=}\right]\right)-\mathrm{k}_{\mathrm{tl}}\left[\lambda_{1}\right] \\
& \left([\mathrm{L} \bullet]+\left[\mathrm{L}^{=} \bullet\right]\right)-\frac{1}{2}\left(\mathrm{k}_{\mathrm{tc}, \mathrm{I}}+\mathrm{k}_{\mathrm{tc}}\right)[\mathrm{I} \bullet]\left[\lambda_{1}\right]-\mathrm{k}_{\mathrm{tc}}\left[\lambda_{0}\right]\left[\lambda_{1}\right]+\mathrm{k}_{\mathrm{p}}\left[\lambda_{0}\right][\mathrm{M}] \\
& \frac{\mathrm{d}\left[\lambda_{2}\right]}{\mathrm{dt}}=-\mathrm{k}_{\mathrm{d}}\left[\lambda_{2}\right]\left[\mathrm{CuBr}_{2}\right]+\mathrm{k}_{\mathrm{a}}\left[\mu_{2}\right][\mathrm{CuBr}]+\mathrm{k}_{\mathrm{p}}[\mathrm{I} \bullet][\mathrm{M}]-\mathrm{k}_{\mathrm{tr}}\left[\lambda_{2}\right]\left([\mathrm{L}]+\left[\mathrm{L}^{=}\right]\right)-\mathrm{k}_{\mathrm{t} 1}\left[\lambda_{2}\right] \\
& \left([\mathrm{L} \bullet]+\left[\mathrm{L}^{=} \bullet\right]\right)-\frac{1}{2}\left(\mathrm{k}_{\mathrm{tc}, \mathrm{I}}+\mathrm{k}_{\mathrm{tc}}\right)[\mathrm{I} \bullet]\left[\lambda_{2}\right]-\mathrm{k}_{\mathrm{tc}}\left[\lambda_{0}\right]\left[\lambda_{2}\right]+\mathrm{k}_{\mathrm{p}}\left[\lambda_{0}\right][\mathrm{M}]+2 \mathrm{k}_{\mathrm{p}}\left[\lambda_{1}\right][\mathrm{M}] \\
& \frac{\mathrm{d}\left[\mu_{0}\right]}{\mathrm{dt}}=\mathrm{k}_{\mathrm{d}}\left[\lambda_{0}\right]\left[\mathrm{CuBr}_{2}\right]-\mathrm{k}_{\mathrm{a}}\left[\mu_{0}\right][\mathrm{CuBr}] \\
& \frac{\mathrm{d}\left[\mu_{1}\right]}{\mathrm{dt}}=\mathrm{k}_{\mathrm{d}}\left[\lambda_{1}\right]\left[\mathrm{CuBr}_{2}\right]-\mathrm{k}_{\mathrm{a}}\left[\mu_{1}\right][\mathrm{CuBr}] \\
& \frac{\mathrm{d}\left[\mu_{2}\right]}{\mathrm{dt}}=\mathrm{k}_{\mathrm{d}}\left[\lambda_{2}\right]\left[\mathrm{CuBr}_{2}\right]-\mathrm{k}_{\mathrm{a}}\left[\mu_{2}\right][\mathrm{CuBr}] \\
& \frac{\mathrm{d}\left[\chi_{0}\right]}{\mathrm{dt}}=\mathrm{k}_{\mathrm{tr}}\left[\lambda_{0}\right]\left([\mathrm{L}]+\left[\mathrm{L}^{=}\right]\right)+\mathrm{k}_{\mathrm{tl}}\left[\lambda_{0}\right]\left([\mathrm{L} \bullet]+\left[\mathrm{L}^{=} \bullet\right]\right)+\frac{1}{2}\left(\mathrm{k}_{\mathrm{tc}, \mathrm{I}}+\mathrm{k}_{\mathrm{tc}}\right)[\mathrm{I} \bullet]\left[\lambda_{0}\right]+\frac{\mathrm{k}_{\mathrm{tc}}}{2}\left[\lambda_{0}\right]^{2} \\
& \frac{\mathrm{d}\left[\chi_{1}\right]}{\mathrm{dt}}=\mathrm{k}_{\mathrm{tr}}\left[\lambda_{1}\right]\left([\mathrm{L}]+\left[\mathrm{L}^{=}\right]\right)+\mathrm{k}_{\mathrm{tl}}\left[\lambda_{1}\right]\left([\mathrm{L} \bullet]+\left[\mathrm{L}^{=} \bullet\right]\right)+\frac{1}{2}\left(\mathrm{k}_{\mathrm{tc}, \mathrm{I}}+\mathrm{k}_{\mathrm{tc}}\right)[\mathrm{I} \bullet]\left[\lambda_{1}\right]+\mathrm{k}_{\mathrm{tc}}\left[\lambda_{0}\right]\left[\lambda_{1}\right]
\end{aligned}
$$




$$
\begin{aligned}
& \frac{\mathrm{d}\left[\chi_{2}\right]}{\mathrm{dt}}=\mathrm{k}_{\mathrm{tr}}\left[\lambda_{2}\right]\left([\mathrm{L}]+\left[\mathrm{L}^{=}\right]\right)+\mathrm{k}_{\mathrm{tl}}\left[\lambda_{2}\right]\left([\mathrm{L} \bullet]+\left[\mathrm{L}^{=} \bullet\right]\right)+\frac{1}{2}\left(\mathrm{k}_{\mathrm{tc}, \mathrm{I}}+\mathrm{k}_{\mathrm{tc}}\right)[\mathrm{I} \bullet]\left[\lambda_{2}\right]+\mathrm{k}_{\mathrm{tc}}\left(\left[\lambda_{0}\right]\left[\lambda_{2}\right]+\left[\lambda_{1}\right]^{2}\right) \\
& \frac{\mathrm{d}[\mathrm{CuBr}]}{\mathrm{dt}}=\mathrm{k}_{\mathrm{d}}\left(\left[\lambda_{0}\right]+[\mathrm{I} \bullet]\right)\left[\mathrm{CuBr}_{2}\right]-\mathrm{k}_{\mathrm{a}}\left(\left[\mu_{0}\right]+[\mathrm{IBr}]\right)[\mathrm{CuBr}] \\
& \frac{\mathrm{d}[\mathrm{M}]}{\mathrm{dt}}=-\mathrm{k}_{\mathrm{p}}\left[\lambda_{0}\right][\mathrm{M}]-\mathrm{k}_{\mathrm{p}}[\mathrm{I} \bullet][\mathrm{M}] \\
& \frac{\mathrm{d}[\mathrm{L}]}{\mathrm{dt}}=-\mathrm{k}_{\mathrm{tr}}\left(\left[\lambda_{0}\right]+[\mathrm{I} \bullet]\right)[\mathrm{L}]+\mathrm{k}_{\mathrm{tl}}\left(\left[\lambda_{0}\right]+[\mathrm{I} \bullet]\right)[\mathrm{L} \bullet]+\mathrm{k}_{\mathrm{tll}}[\mathrm{L} \bullet]\left([\mathrm{L} \bullet]+\left[\mathrm{L}^{=} \bullet\right]\right) \\
& \frac{\mathrm{d}[\mathrm{L} \bullet]}{\mathrm{dt}}=\mathrm{k}_{\mathrm{tr}}\left(\left[\lambda_{0}\right]+[\mathrm{I} \bullet]\right)[\mathrm{L}]-\mathrm{k}_{\mathrm{tl}}\left(\left[\lambda_{0}\right]+[\mathrm{I} \bullet]\right)[\mathrm{L} \bullet]-\mathrm{k}_{\mathrm{tll}}[\mathrm{L} \bullet]\left(2[\mathrm{~L} \bullet]+\left[\mathrm{L}^{=} \bullet\right]\right) \\
& \frac{\mathrm{d}\left[\mathrm{L}^{=}\right]}{\mathrm{dt}}=-\mathrm{k}_{\mathrm{tr}}\left(\left[\lambda_{0}\right]+[\mathrm{I} \bullet]\right)\left[\mathrm{L}^{=}\right]+\mathrm{k}_{\mathrm{tl}}\left(\left[\lambda_{0}\right]+[\mathrm{I} \bullet]\right)\left[\mathrm{L}^{=} \bullet\right]+\mathrm{k}_{\mathrm{tll}}[\mathrm{L} \bullet]\left([\mathrm{L} \bullet]+\left[\mathrm{L}^{=} \bullet\right]\right)+\mathrm{k}_{\mathrm{tll}}\left[\mathrm{L}^{=} \bullet\right]^{2} \\
& \frac{\mathrm{d}\left[\mathrm{L}^{=} \bullet\right]}{\mathrm{dt}}=\mathrm{k}_{\mathrm{tr}}\left(\left[\lambda_{0}\right]+[\mathrm{I} \bullet]\right)\left[\mathrm{L}^{=}\right]-\mathrm{k}_{\mathrm{tl}}\left(\left[\lambda_{0}\right]+[\mathrm{I} \bullet]\right)\left[\mathrm{L}^{=} \bullet\right]-\mathrm{k}_{\mathrm{tll}}\left[\mathrm{L}^{=} \bullet\right]\left([\mathrm{L} \bullet]+\left[\mathrm{L}^{=} \bullet\right]\right) \\
& \frac{\mathrm{d}\left[\mathrm{L}_{=}^{=}\right]}{\mathrm{dt}}=\mathrm{k}_{\mathrm{tll}}\left(\frac{1}{2}[\mathrm{~L} \bullet]+\left[\mathrm{L}^{=} \bullet\right]\right)\left[\mathrm{L}^{=} \bullet\right]
\end{aligned}
$$

Section 3: Kinetic balance equations for modeling the ATRP of nBA on the basis of the proposed ATRP mechanism that includes the chain transfer to ligand and the difference in reactivity between the free and copper-bound ligand species.

$$
\begin{aligned}
& \frac{\mathrm{d}[\mathrm{IBr}]}{\mathrm{dt}}=\mathrm{k}_{\mathrm{d}}[\mathrm{I} \bullet]\left[\mathrm{CuBr}_{2}\right]-\mathrm{k}_{\mathrm{a}}[\mathrm{IBr}][\mathrm{CuBr}] \\
& \frac{\mathrm{d}[\mathrm{I} \bullet]}{\mathrm{dt}}=-\mathrm{k}_{\mathrm{d}}[\mathrm{I} \bullet]\left[\mathrm{CuBr}_{2}\right]+\mathrm{k}_{\mathrm{a}}[\mathrm{IBr}][\mathrm{CuBr}]-\mathrm{k}_{\mathrm{p}}[\mathrm{I} \bullet][\mathrm{M}]-\mathrm{k}_{\mathrm{tr}}^{\prime}[\mathrm{I} \bullet]\left([\mathrm{CuL}]+\left[\mathrm{CuL}^{=}\right]\right)-\mathrm{k}_{\mathrm{tr}}[\mathrm{I} \bullet] \\
& \left([\mathrm{L}]+\left[\mathrm{L}^{=}\right]\right)-\mathrm{k}_{\mathrm{t} 1 \mathrm{~L}}^{\prime}[\mathrm{I} \bullet]\left([\mathrm{CuL} \bullet]+\left[\mathrm{CuL}^{=} \bullet\right]\right)-\mathrm{k}_{\mathrm{tl}}[\mathrm{I} \bullet]\left([\mathrm{L} \bullet]+\left[\mathrm{L}^{=} \bullet\right]\right)-\frac{1}{2}\left(\mathrm{k}_{\mathrm{tc}, \mathrm{I}}+\mathrm{k}_{\mathrm{tc}}\right)[\mathrm{I} \bullet]\left[\lambda_{0}\right]- \\
& \mathrm{k}_{\mathrm{tc}, \mathrm{I}}[\mathrm{I} \bullet]^{2} \\
& \frac{\mathrm{d}\left[\lambda_{0}\right]}{\mathrm{dt}}=-\mathrm{k}_{\mathrm{d}}\left[\lambda_{0}\right]\left[\mathrm{CuBr}_{2}\right]+\mathrm{k}_{\mathrm{a}}\left[\mu_{0}\right][\mathrm{CuBr}]+\mathrm{k}_{\mathrm{p}}[\mathrm{I} \bullet][\mathrm{M}]-\mathrm{k}_{\mathrm{tr}}^{\prime}\left[\lambda_{0}\right]\left([\mathrm{CuL}]+\left[\mathrm{CuL}^{=}\right]\right)-\mathrm{k}_{\mathrm{tr}}\left[\lambda_{0}\right] \\
& \left([\mathrm{L}]+\left[\mathrm{L}^{=}\right]\right)-\mathrm{k}_{\mathrm{tl}}^{\prime}\left[\lambda_{0}\right]\left([\mathrm{CuL} \bullet]+\left[\mathrm{CuL}^{=} \bullet\right]\right)-\mathrm{k}_{\mathrm{tl}}\left[\lambda_{0}\right]\left([\mathrm{L} \bullet]+\left[\mathrm{L}^{=} \bullet\right]\right)-\frac{1}{2}\left(\mathrm{k}_{\mathrm{tc}, \mathrm{I}}+\mathrm{k}_{\mathrm{tc}}\right)[\mathrm{I} \bullet]\left[\lambda_{0}\right]- \\
& \mathrm{k}_{\mathrm{tc}}\left[\lambda_{0}\right]^{2} \\
& \frac{\mathrm{d}\left[\lambda_{1}\right]}{\mathrm{dt}}=-\mathrm{k}_{\mathrm{d}}\left[\lambda_{1}\right]\left[\mathrm{CuBr}_{2}\right]+\mathrm{k}_{\mathrm{a}}\left[\mu_{1}\right][\mathrm{CuBr}]+\mathrm{k}_{\mathrm{p}}[\mathrm{I} \bullet][\mathrm{M}]-\mathrm{k}_{\mathrm{tr}}^{\prime}\left[\lambda_{1}\right]\left([\mathrm{CuL}]+\left[\mathrm{CuL}={ }^{=}\right]\right)-\mathrm{k}_{\mathrm{tr}}\left[\lambda_{1}\right] \\
& \left([\mathrm{L}]+\left[\mathrm{L}^{=}\right]\right)-\mathrm{k}_{\mathrm{tl}}^{\prime}\left[\lambda_{1}\right]\left([\mathrm{CuL} \bullet]+\left[\mathrm{CuL}^{=} \bullet\right]\right)-\mathrm{k}_{\mathrm{tl}}\left[\lambda_{1}\right]\left([\mathrm{L} \bullet]+\left[\mathrm{L}^{=} \bullet\right]\right)-\frac{1}{2}\left(\mathrm{k}_{\mathrm{tc}, \mathrm{I}}+\mathrm{k}_{\mathrm{tc}}\right)[\mathrm{I} \bullet]\left[\lambda_{1}\right]- \\
& \mathrm{k}_{\mathrm{tc}}\left[\lambda_{0}\right]\left[\lambda_{1}\right]+\mathrm{k}_{\mathrm{p}}\left[\lambda_{0}\right][\mathrm{M}]
\end{aligned}
$$




$$
\begin{aligned}
& \frac{\mathrm{d}\left[\lambda_{2}\right]}{\mathrm{dt}}=-\mathrm{k}_{\mathrm{d}}\left[\lambda_{2}\right]\left[\mathrm{CuBr}_{2}\right]+\mathrm{k}_{\mathrm{a}}\left[\mu_{2}\right][\mathrm{CuBr}]+\mathrm{k}_{\mathrm{p}}[\mathrm{I} \bullet][\mathrm{M}]-\mathrm{k}_{\mathrm{trr}}^{\prime}\left[\lambda_{2}\right]\left([\mathrm{CuL}]+\left[\mathrm{CuL}^{=}\right]\right)-\mathrm{k}_{\mathrm{tr}}\left[\lambda_{2}\right] \\
& \left([\mathrm{L}]+\left[\mathrm{L}^{=}\right]\right)-\mathrm{k}_{\mathrm{tt}}^{\prime}\left[\lambda_{2}\right]\left([\mathrm{CuL} \bullet]+\left[\mathrm{CuL}^{=} \bullet\right]\right)-\mathrm{k}_{\mathrm{tt}}\left[\lambda_{2}\right]\left([\mathrm{L} \bullet]+\left[\mathrm{L}^{=} \bullet\right]\right)-\frac{1}{2}\left(\mathrm{k}_{\mathrm{tc}, \mathrm{I}}+\mathrm{k}_{\mathrm{tc}}\right)\left[\boldsymbol{l}_{\bullet}\right]\left[\lambda_{2}\right]- \\
& \mathrm{k}_{\mathrm{tc}}\left[\lambda_{0}\right]\left[\lambda_{2}\right]+\mathrm{k}_{\mathrm{p}}\left[\lambda_{0}\right][\mathrm{M}]+2 \mathrm{k}_{\mathrm{p}}\left[\lambda_{1}\right][\mathrm{M}] \\
& \frac{\mathrm{d}\left[\mu_{0}\right]}{\mathrm{dt}}=\mathrm{k}_{\mathrm{d}}\left[\lambda_{0}\right]\left[\mathrm{CuBr}_{2}\right]-\mathrm{k}_{\mathrm{a}}\left[\mu_{0}\right][\mathrm{CuBr}] \\
& \frac{\mathrm{d}\left[\mu_{1}\right]}{\mathrm{dt}}=\mathrm{k}_{\mathrm{d}}\left[\lambda_{1}\right]\left[\mathrm{CuBr}_{2}\right]-\mathrm{k}_{\mathrm{a}}\left[\mu_{1}\right][\mathrm{CuBr}] \\
& \frac{\mathrm{d}\left[\mu_{2}\right]}{\mathrm{dt}}=\mathrm{k}_{\mathrm{d}}\left[\lambda_{2}\right]\left[\mathrm{CuBr}_{2}\right]-\mathrm{k}_{\mathrm{a}}\left[\mu_{2}\right][\mathrm{CuBr}] \\
& \frac{\mathrm{d}\left[\chi_{0}\right]}{\mathrm{dt}}=\mathrm{k}_{\mathrm{tr}}^{\prime}\left[\lambda_{0}\right]\left([\mathrm{CuL}]+\left[\mathrm{CuL}^{=}\right]\right)+\mathrm{k}_{\mathrm{tr}}\left[\lambda_{0}\right]\left([\mathrm{L}]+\left[\mathrm{L}^{=}\right]\right)+\mathrm{k}_{\mathrm{tt}}^{\prime}\left[\lambda_{0}\right]\left([\mathrm{CuL} \bullet]+\left[\mathrm{CuL}^{=} \bullet\right]\right)+\mathrm{k}_{\mathrm{tl}}\left[\lambda_{0}\right] \\
& \left([\mathrm{L} \bullet]+\left[\mathrm{L}^{=} \bullet\right]\right)+\frac{1}{2}\left(\mathrm{k}_{\mathrm{tc}, \mathrm{I}}+\mathrm{k}_{\mathrm{tc}}\right)[\mathrm{I} \bullet]\left[\lambda_{0}\right]+\frac{\mathrm{k}_{\mathrm{tc}}}{2}\left[\lambda_{0}\right]^{2} \\
& \frac{\mathrm{d}\left[\chi_{1}\right]}{\mathrm{dt}}=\mathrm{k}_{\mathrm{tr}}^{\prime}\left[\lambda_{1}\right]\left([\mathrm{CuL}]+\left[\mathrm{CuL}^{=}\right]\right)+\mathrm{k}_{\mathrm{tr}}\left[\lambda_{1}\right]\left([\mathrm{L}]+\left[\mathrm{L}^{=}\right]\right)+\mathrm{k}_{\mathrm{tt}}^{\prime}\left[\lambda_{1}\right]\left([\mathrm{CuL} \bullet]+\left[\mathrm{CuL}^{=} \bullet\right]\right)+\mathrm{k}_{\mathrm{tt}}\left[\lambda_{1}\right] \\
& \left([\mathrm{L} \bullet]+\left[\mathrm{L}^{=} \bullet\right]\right)+\frac{1}{2}\left(\mathrm{k}_{\mathrm{tc}, \mathrm{I}}+\mathrm{k}_{\mathrm{tc}}\right)[\mathrm{I} \bullet]\left[\lambda_{1}\right]+\mathrm{k}_{\mathrm{tc}}\left[\lambda_{0}\right]\left[\lambda_{1}\right] \\
& \frac{\mathrm{d}\left[\chi_{2}\right]}{\mathrm{dt}}=\mathrm{k}_{\mathrm{tr}}^{\prime}\left[\lambda_{2}\right]\left([\mathrm{CuL}]+\left[\mathrm{CuL}^{=}\right]\right)+\mathrm{k}_{\mathrm{tr}}\left[\lambda_{2}\right]\left([\mathrm{L}]+\left[\mathrm{L}^{=}\right]\right)+\mathrm{k}_{\mathrm{tl}}^{\prime}\left[\lambda_{2}\right]\left([\mathrm{CuL} \bullet]+\left[\mathrm{CuL}^{=} \bullet\right]\right)+\mathrm{k}_{\mathrm{tl}}\left[\lambda_{2}\right] \\
& \left([\mathrm{L} \bullet]+\left[\mathrm{L}^{=} \bullet\right]\right)+\frac{1}{2}\left(\mathrm{k}_{\mathrm{tc}, \mathrm{I}}+\mathrm{k}_{\mathrm{tc}}\right)[\mathrm{I} \bullet]\left[\lambda_{2}\right]+\mathrm{k}_{\mathrm{tc}}\left(\left[\lambda_{0}\right]\left[\lambda_{2}\right]+\left[\lambda_{1}\right]^{2}\right) \\
& \frac{\mathrm{d}[\mathrm{CuBr}]}{\mathrm{dt}}=\mathrm{k}_{\mathrm{d}}\left(\left[\lambda_{0}\right]+\left[\mathrm{I}_{\bullet}\right]\right)\left[\mathrm{CuBr}_{2}\right]-\mathrm{k}_{\mathrm{a}}\left(\left[\mu_{0}\right]+[\mathrm{IBr}]\right)[\mathrm{CuBr}] \\
& \frac{\mathrm{d}[\mathrm{M}]}{\mathrm{dt}}=-\mathrm{k}_{\mathrm{p}}\left[\lambda_{0}\right][\mathrm{M}]-\mathrm{k}_{\mathrm{p}}[\bullet \bullet[\mathrm{M}] \\
& \frac{\mathrm{d}[\mathrm{CuL}]}{\mathrm{dt}}=-\mathrm{k}_{\mathrm{tr}}^{\prime}\left(\left[\lambda_{0}\right]+[\mathrm{I} \bullet]\right)[\mathrm{CuL}]+\mathrm{k}_{\mathrm{tl}}^{\prime}\left(\left[\lambda_{0}\right]+[\mathrm{I} \bullet]\right)[\mathrm{CuL} \bullet]+\mathrm{k}_{\mathrm{ttl}}^{\prime}[\mathrm{CuL} \bullet]\left([\mathrm{CuL} \bullet]+\frac{1}{2}\left[\mathrm{CuL}^{=} \bullet\right]\right) \\
& +\frac{1}{2} \mathrm{k}_{\mathrm{ttll}}^{\prime \prime}[\mathrm{CuL} \bullet]\left([\mathrm{L} \bullet]+\left[\mathrm{L}^{=} \bullet\right]\right) \\
& \frac{\mathrm{d}[\mathrm{L}]}{\mathrm{dt}}=-\mathrm{k}_{\mathrm{tr}}\left(\left[\lambda_{0}\right]+[\mathrm{I} \bullet]\right)[\mathrm{L}]+\mathrm{k}_{\mathrm{tl}}\left(\left[\lambda_{0}\right]+[\mathrm{I} \bullet]\right)[\mathrm{L} \bullet]+\mathrm{k}_{\mathrm{tll}}[\mathrm{L} \bullet]\left([\mathrm{L} \bullet]+\frac{1}{2}\left[\mathrm{~L}^{=} \bullet\right]\right)+\frac{1}{2} \mathrm{k}_{\mathrm{tll}}^{\prime \prime}[\mathrm{L} \bullet] \\
& \left([\mathrm{CuL} \bullet]+\left[\mathrm{CuL}^{=} \bullet\right]\right) \\
& \frac{\mathrm{d}[\mathrm{CuL} \bullet}{\mathrm{dt}}=\mathrm{k}_{\mathrm{tr}}^{\prime}\left(\left[\lambda_{0}\right]+[\mathrm{I} \bullet]\right)[\mathrm{CuL}]-\mathrm{k}_{\mathrm{tl}}^{\prime}\left(\left[\lambda_{0}\right]+[\mathrm{I} \bullet]\right)[\mathrm{CuL} \bullet]-\mathrm{k}_{\mathrm{tIl}}^{\prime}[\mathrm{CuL} \bullet]\left(2[\mathrm{CuL} \bullet]+\left[\mathrm{CuL}^{=} \bullet\right]\right) \\
& -\mathrm{k}_{\mathrm{tIl}}^{\mathrm{\prime l}}[\mathrm{CuL} \bullet]\left([\mathrm{L} \bullet]+\left[\mathrm{L}^{=} \bullet\right]\right) \\
& \frac{\mathrm{d}[\mathrm{L} \bullet}{\mathrm{dt}}=\mathrm{k}_{\mathrm{tr}}\left(\left[\lambda_{0}\right]+[\mathrm{I} \bullet]\right)[\mathrm{L}]-\mathrm{k}_{\mathrm{tl}}\left(\left[\lambda_{0}\right]+[\mathrm{\bullet} \bullet]\right)[\mathrm{L} \bullet]-\mathrm{k}_{\mathrm{tll}}[\mathrm{L} \bullet]\left(2[\mathrm{~L} \bullet]+\left[\mathrm{L}^{=} \bullet\right]\right)-\mathrm{k}_{\mathrm{ttl}}^{\prime \prime}[\mathrm{L} \bullet]([\mathrm{CuL} \bullet \\
& \left.+\left[\mathrm{L}^{=} \bullet\right]\right)
\end{aligned}
$$




$$
\begin{aligned}
& \frac{\mathrm{d}\left[\mathrm{CuL}^{=}\right]}{\mathrm{dt}}=-\mathrm{k}_{\mathrm{tr}}^{\prime}\left(\left[\lambda_{0}\right]+\left[\mathrm{I}_{\bullet}\right]\right)\left[\mathrm{CuL}^{=}\right]+\mathrm{k}_{\mathrm{tl}}^{\prime}\left(\left[\lambda_{0}\right]+[\mathrm{I} \bullet]\right)\left[\mathrm{CuL}^{=} \bullet\right]+\mathrm{k}_{\mathrm{tll}}^{\prime}[\mathrm{CuL} \bullet]\left([\mathrm{CuL} \bullet]+\left[\mathrm{CuL}^{=} \bullet\right]\right) \\
& +\mathrm{k}_{\mathrm{tll}}^{\prime}\left[\mathrm{CuL}^{=} \bullet\right]^{2}+\frac{1}{2} \mathrm{k}_{\mathrm{tIll}}^{\mathrm{\prime l}}[\mathrm{CuL} \bullet]\left([\mathrm{L} \bullet]+\left[\mathrm{L}^{=} \bullet\right]\right)+\frac{1}{2} \mathrm{k}_{\mathrm{tll}}^{\prime \prime}[\mathrm{L} \bullet]\left[\mathrm{CuL}^{=} \bullet\right]+\frac{1}{2} \mathrm{k}_{\mathrm{tll}}^{\prime \prime}\left[\mathrm{CuL}^{=} \bullet\right] \\
& {\left[\mathrm{L}^{=} \bullet\right]} \\
& \frac{\mathrm{d}\left[\mathrm{L}^{=}\right]}{\mathrm{dt}}=-\mathrm{k}_{\mathrm{tr}}\left(\left[\lambda_{0}\right]+[\mathrm{l} \bullet]\right)\left[\mathrm{L}^{=}\right]+\mathrm{k}_{\mathrm{tl}}\left(\left[\lambda_{0}\right]+[\mathrm{I} \bullet]\right)\left[\mathrm{L}^{=} \bullet\right]+\mathrm{k}_{\mathrm{tll}}[\mathrm{L} \bullet]\left([\mathrm{L} \bullet]+\left[\mathrm{L}^{=} \bullet\right]\right)+\mathrm{k}_{\mathrm{tll}}\left[\mathrm{L}^{=} \bullet\right]^{2}+\frac{1}{2} \\
& \mathrm{k}_{\mathrm{tll}}^{\prime \prime}[\mathrm{L} \bullet]\left([\mathrm{CuL} \bullet]+\left[\mathrm{CuL}^{=} \bullet\right]\right)+\frac{1}{2} \mathrm{k}_{\mathrm{tll}}^{\mathrm{tl}}[\mathrm{CuL} \bullet]\left[\mathrm{L}^{=} \bullet\right]+\frac{1}{2} \mathrm{k}_{\mathrm{tll}}^{\prime \prime}\left[\mathrm{L}^{=} \bullet\right]\left[\mathrm{CuL}^{=} \bullet\right] \\
& \frac{\mathrm{d}\left[\mathrm{CuL}^{=} \bullet\right]}{\mathrm{dt}}=\mathrm{k}_{\mathrm{tr}}^{\prime}\left(\left[\lambda_{0}\right]+[\mathrm{I} \bullet]\right)\left[\mathrm{CuL}^{=}\right]-\mathrm{k}_{\mathrm{tl}}^{\prime}\left(\left[\lambda_{0}\right]+[\mathrm{I} \bullet]\right)\left[\mathrm{CuL}^{=} \bullet\right]-\mathrm{k}_{\mathrm{tll}}^{\prime}\left[\mathrm{CuL}^{=} \bullet\right]\left([\mathrm{CuL} \bullet]+\left[\mathrm{CuL}^{=} \bullet\right]\right) \\
& -\mathrm{k}_{\mathrm{tII}}^{\prime \prime}\left[\mathrm{L}^{=} \bullet\right]\left([\mathrm{CuL} \bullet]+\left[\mathrm{CuL}^{=} \bullet\right]\right) \\
& \frac{\mathrm{d}\left[\mathrm{L}^{=} \bullet\right]}{\mathrm{dt}}=\mathrm{k}_{\mathrm{tr}}\left(\left[\lambda_{0}\right]+[\mathrm{I} \bullet]\right)\left[\mathrm{L}^{=}\right]-\mathrm{k}_{\mathrm{tl}}\left(\left[\lambda_{0}\right]+[\mathrm{I} \bullet]\right)\left[\mathrm{L}^{=} \bullet\right]-\mathrm{k}_{\mathrm{tll}}\left[\mathrm{L}^{=} \bullet\right]\left([\mathrm{L} \bullet]+\left[\mathrm{L}^{=} \bullet\right]\right)-\mathrm{k}_{\mathrm{ttl}}^{\prime \prime}\left[\mathrm{CuL}^{=} \bullet\right] \\
& \left([\mathrm{L} \bullet]+\left[\mathrm{L}^{=} \bullet\right]\right) \\
& \frac{\mathrm{d}\left[\mathrm{CuL}_{=}^{=}\right]}{\mathrm{dt}}=\mathrm{k}_{\mathrm{tll}}^{\prime}\left(\frac{1}{2}[\mathrm{CuL} \bullet]+\left[\mathrm{CuL}^{=} \bullet\right)\left[\mathrm{CuL}^{=} \bullet\right]+\frac{1}{2} \mathrm{k}_{\mathrm{tII}}^{\prime \prime}\left[\mathrm{CuL}^{=} \bullet\right]\left([\mathrm{L} \bullet]+\left[\mathrm{L}^{=} \bullet\right]\right)\right. \\
& \frac{\mathrm{d}\left[\mathrm{L}_{=}^{\equiv}\right]}{\mathrm{dt}}=\mathrm{k}_{\mathrm{tll}}\left(\frac{1}{2}\left[\mathrm{~L} \bullet_{\bullet}\right]+\left[\mathrm{L}^{=} \bullet\right]\right)\left[\mathrm{L}^{=} \bullet\right]+\frac{1}{2} \mathrm{k}_{\text {tll }}^{\prime \prime}\left[\mathrm{L}^{=} \bullet\right]\left([\mathrm{CuL} \bullet]+\left[\mathrm{CuL}^{=} \bullet\right]\right) \\
& \mathrm{k}_{\mathrm{tr}}^{\prime}=\frac{\mathrm{k}_{\mathrm{tr}}}{\alpha} \\
& \mathrm{k}_{\mathrm{tl}}^{\prime}=\alpha \mathrm{k}_{\mathrm{tl}} \\
& \mathrm{k}_{\mathrm{tll}}^{\prime}=\alpha^{2} \mathrm{k}_{\mathrm{tll}} \\
& \mathrm{k}_{\mathrm{tll}}^{\prime \prime}=\alpha \mathrm{k}_{\mathrm{tll}}
\end{aligned}
$$

\section{Section 4: Procedures for calculating the absolute PDI values for the PEO-PnBA}

product.

The time-dependent molecular weight distribution of the ATRP product (i.e., PEO-PnBA diblock copolymer) can be considered in terms of the populations of chains of all possible compositions for a given overall chain length $\mathrm{n}$ at time $\mathrm{t}$ :

$$
p_{P E O-P n B A}(n, t)=(1-\beta(t)) \sum_{n_{P E O}=1}^{n-1} p_{P E O}\left(n_{P E O}\right) p_{P n B A}\left(n-n_{P E O}, t\right)+\beta(t) p_{P E O}(n)
$$

where $p_{\text {PEO-PnBA }}(n, t), p_{\text {PEO }}(n)$, and $p_{\text {PnBA }}(n, t)$ are the number fractions of chains of length $\mathrm{n}$ within the individual categories of PEO-PnBA, PEO and PnBA, respectively, and $\beta$ is defined as $\beta \equiv\left([\mathrm{IBr}]+\left[\mathrm{I}_{\bullet}\right]+\left[\mathrm{P}_{0}\right]\right) /\left([\mathrm{IBr}]+[\mathrm{I} \bullet]+\left[\mathrm{P}_{0}\right]+\left[\lambda_{0}\right]+\left[\mu_{0}\right]+\left[\chi_{0}\right]\right)$. Starting from this expression, it is not difficult to show that the zeroth, first and second moments 
$\left(\mu_{0}^{\text {PEO-PnBA }}, \mu_{1}^{\text {PEO-PnBA }}\right.$ and $\mu_{2}^{\text {PEO-PnBA }}$, respectively) of the distribution $p_{\text {PEO-PnBA }}(n, t)$ at time $t$ can be written as:

$$
\begin{gathered}
\mu_{0}^{\mathrm{PEO}-\mathrm{PnBA}}=(1-\beta) \mu_{0}^{\mathrm{PEO}} \mu_{0}^{\mathrm{PnBA}}+\beta \mu_{0}^{\mathrm{PEO}} ; \\
\mu_{1}^{\mathrm{PEO}-\mathrm{PnBA}}=(1-\beta)\left(\mu_{0}^{\mathrm{PEO}} \mu_{1}^{\mathrm{PnBA}}+\mu_{1}^{\mathrm{PEO}} \mu_{0}^{\mathrm{PnBA}}\right)+\beta \mu_{1}^{\mathrm{PEO}} ; \\
\mu_{2}^{\mathrm{PEO}-\mathrm{PnBA}}=(1-\beta)\left(\mu_{0}^{\mathrm{PEO}} \mu_{2}^{\mathrm{PnBA}}+2 \mu_{1}^{\mathrm{PEO}} \mu_{1}^{\mathrm{PBA}}+\mu_{2}^{\mathrm{PEO}} \mu_{0}^{\mathrm{PnBA}}\right)+\beta \mu_{2}^{\mathrm{PEO}} .
\end{gathered}
$$

Once the diblock moments are known, the number- and weight-average degrees of polymerization and the PDI for the PEO-PnBA product can be evaluated using:

$$
\begin{gathered}
\overline{\mathrm{DP}}_{\mathrm{n}}^{\mathrm{PEO}-\mathrm{PnBA}}=\frac{\mu_{1}^{\mathrm{PEO}-\mathrm{PnBA}}}{\mu_{0}^{\mathrm{PEO}-\mathrm{PnBA}}} ; \\
\overline{\mathrm{DP}}_{\mathrm{w}}^{\mathrm{PEO}-\mathrm{PnBA}}=\frac{\mu_{2}^{\mathrm{PEO}-\mathrm{PnBA}}}{\mu_{1}^{\mathrm{PEO}-\mathrm{PnBA}}} ; \\
\mathrm{PDI}=\frac{\overline{\mathrm{DP}}_{\mathrm{w}}^{\mathrm{PEO}-\mathrm{PnBA}}}{\overline{\mathrm{DP}}_{\mathrm{n}}^{\mathrm{PEO}-\mathrm{PnBA}}} .
\end{gathered}
$$

In this work, the zeroth, first and second moments of the molecular weight distributions for the individual blocks (i.e., $\mu_{i}^{\text {PEO }}$ and $\mu_{i}^{\text {PnBA }}$ with $i=0,1,2$ ) were computed from estimates of $\mathrm{p}_{\mathrm{PEO}}(\mathrm{n})$ and $\mathrm{p}_{\mathrm{PBA}}(\mathrm{n}, \mathrm{t})$ using the continuum approximation as described below.

The PEO-block distribution $\mathrm{p}_{\mathrm{PEO}}(\mathrm{n})$ was estimated from the GPC trace of the PEO macroinitiator; the experimental GPC data were fit to the model

$$
\mathrm{p}_{\mathrm{PEO}}(\mathrm{n})=\frac{\mathrm{w}}{\sqrt{2 \pi} \sigma_{1}} \mathrm{e}^{-\frac{\left(\mathrm{n}-\overline{\mathrm{n}_{1}}\right)^{2}}{2 \sigma_{1}^{2}}}+\frac{(1-\mathrm{w})}{\sqrt{2 \pi} \sigma_{2}} \mathrm{e}^{-\frac{\left(\mathrm{n}-\overline{\mathrm{n}_{2}}\right)^{2}}{2 \sigma_{2}^{2}}} .
$$

Here the use of two Gaussian distribution functions was necessitated by the reproducible occurrence of a small shoulder at the high molecular weight side of the GPC profile.

In doing the above analysis, the PEO molecular weights determined by GPC on the basis of polystyrene standard samples were converted into absolute molecular weight values using the following relation

$$
\mathrm{M}_{\mathrm{PEO}}=\left(\frac{\mathrm{K}_{\mathrm{PS}}}{\mathrm{K}_{\mathrm{PEO}}} \mathrm{M}_{\mathrm{PS}}{ }^{\left(1+\alpha_{\mathrm{PS}}\right)}\right)^{\frac{1}{\left(1+\alpha_{\mathrm{PEO}}\right)}}
$$

where $K_{x}$ and $\alpha_{x}$ are the Mark-Houwink prefactor and exponent, respectively, for polymer of type $x$ in THF.

Assuming that the chain length distribution of the PnBA block also follows a Gaussian distribution, $\mathrm{p}_{\mathrm{PBA}}(\mathrm{n}, \mathrm{t})$ can be written as

$$
\mathrm{p}_{\mathrm{PBA}}(\mathrm{n}, \mathrm{t})=\frac{1}{\sqrt{2 \pi} \sigma} \mathrm{e}^{-\frac{(\mathrm{n}-\overline{\mathrm{n}})^{2}}{2 \sigma^{2}}} \text {. }
$$


The mean $\overline{\mathrm{n}}(\mathrm{t})$ and the standard deviation $\sigma(\mathrm{t})$ of this distribution can be evaluated from the kinetic modeling results:

$$
\begin{gathered}
\overline{\mathrm{n}}(\mathrm{t})=\frac{\left[\lambda_{1}\right]+\left[\mu_{1}\right]+\left[\chi_{1}\right]}{\left[\lambda_{0}\right]+\left[\mu_{0}\right]+\left[\chi_{0}\right]} ; \\
\sigma(\mathrm{t})=\sqrt{\frac{\left[\lambda_{2}\right]+\left[\mu_{2}\right]+\left[\chi_{2}\right]}{\left[\lambda_{0}\right]+\left[\mu_{0}\right]+\left[\chi_{0}\right]}-\overline{\mathrm{n}}(\mathrm{t})^{2}} .
\end{gathered}
$$

Section 5: Sensitivity of the predictions of the proposed ATRP model to variations in the kinetic parameters (see Figures S1 (a) through (f) below).

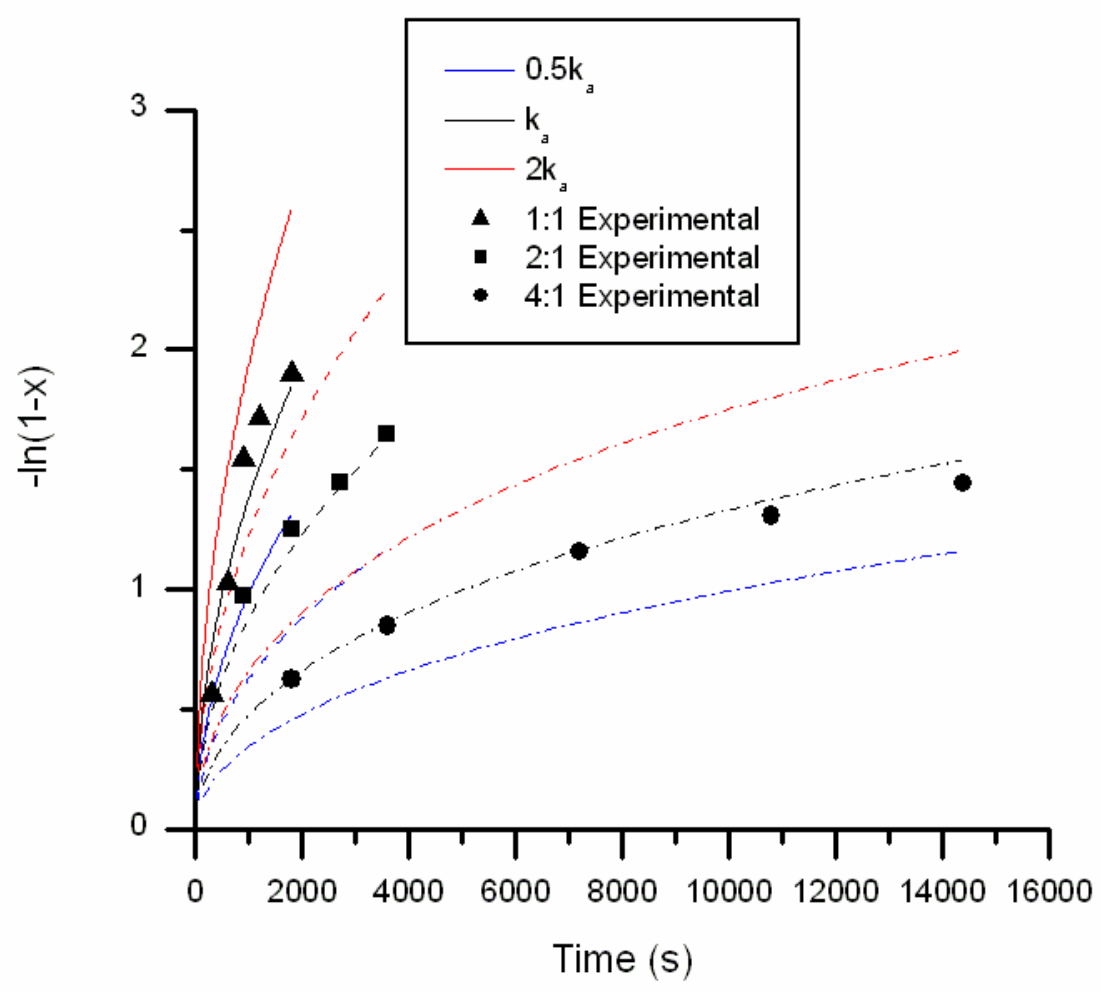

(a) 


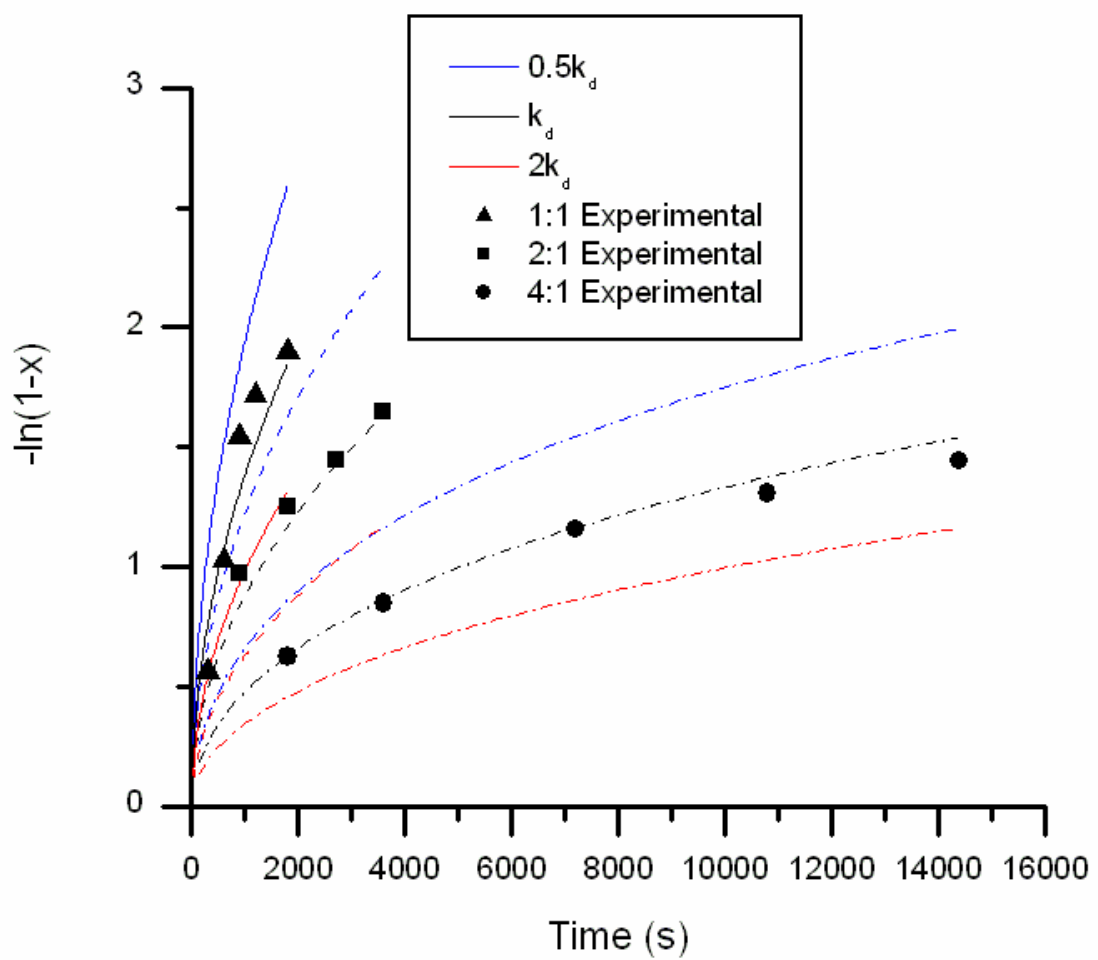

(b)

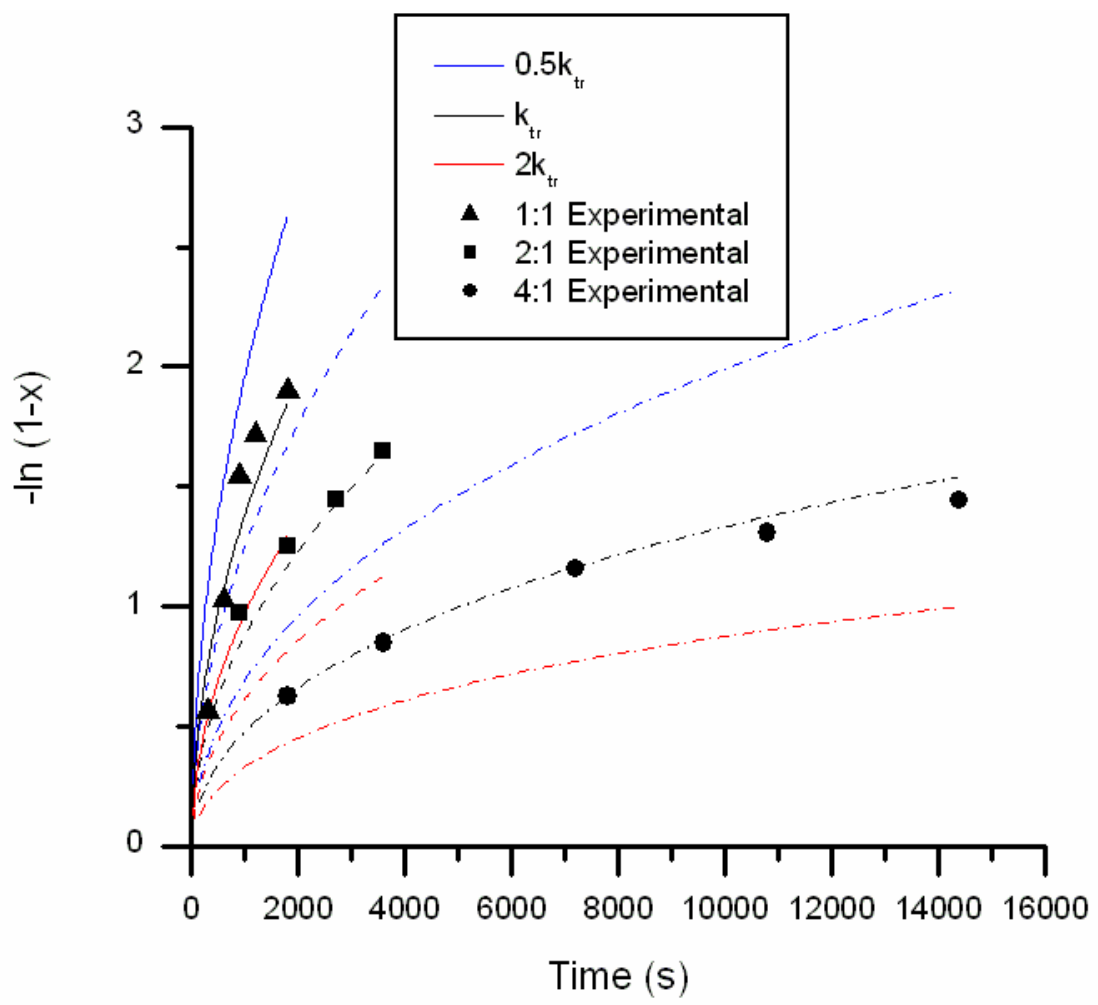

(c) 


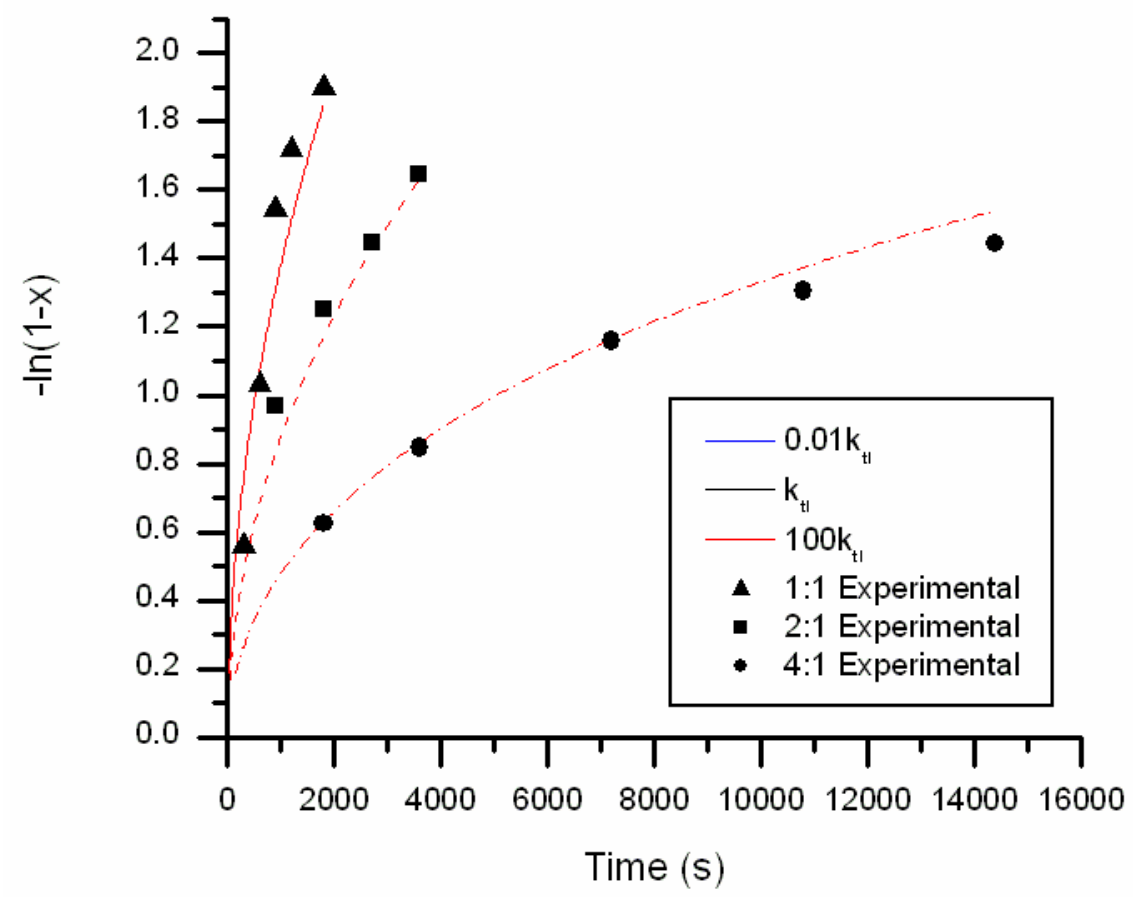

(d)

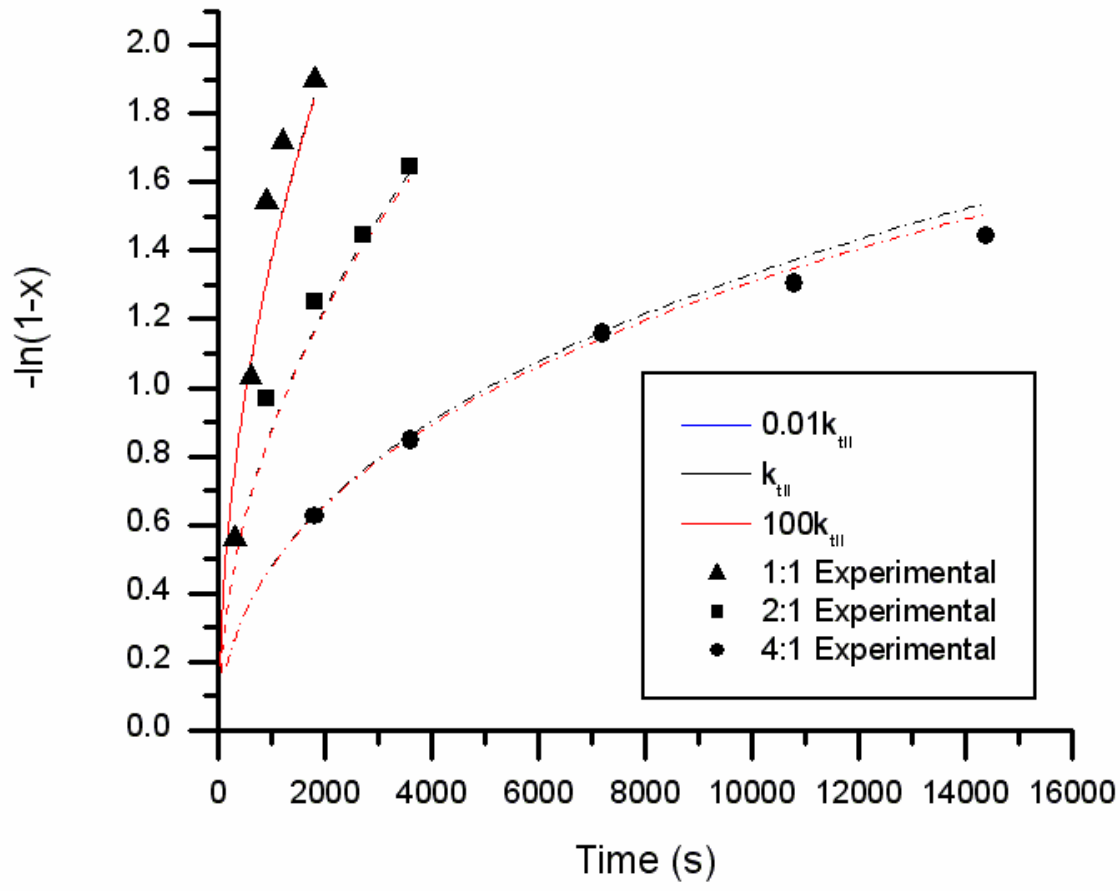

(e) 


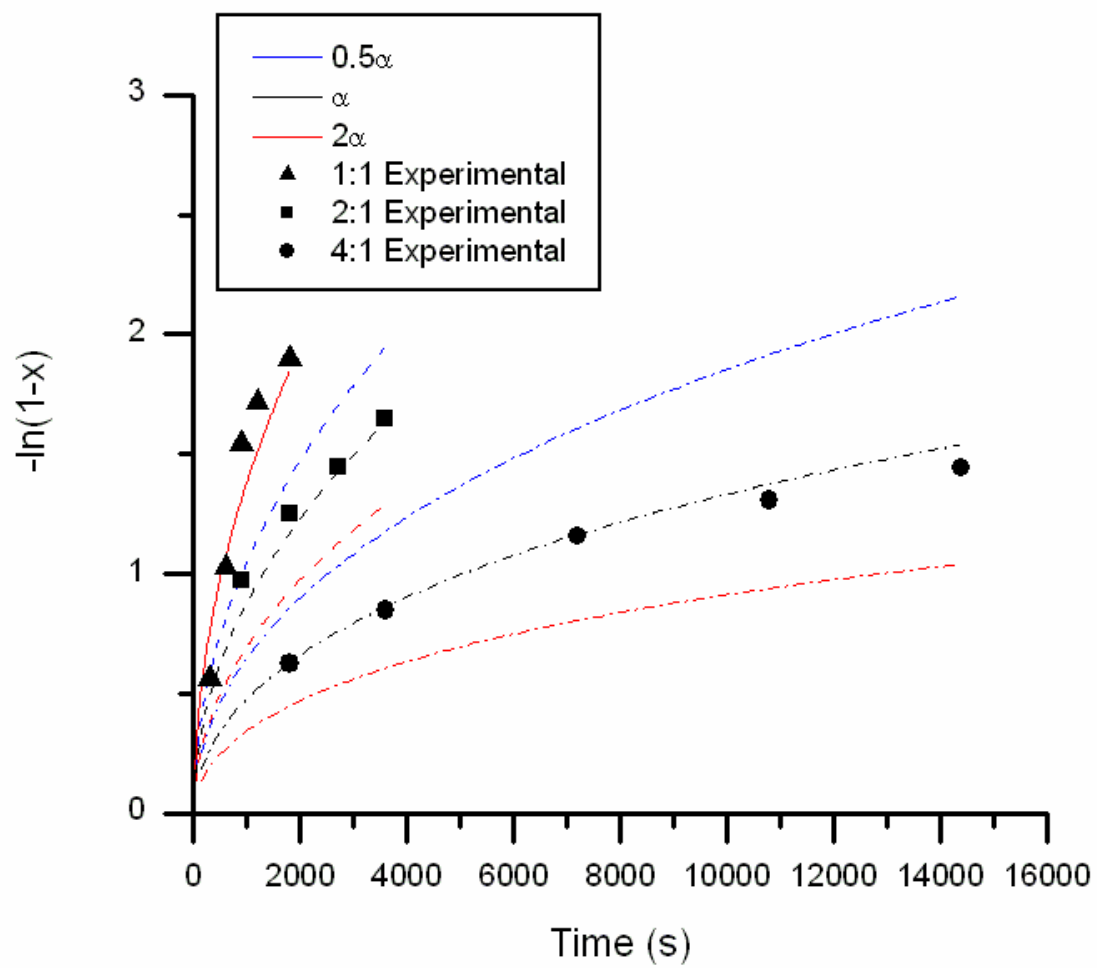

(f)

Figure S1. Demonstrations of how a factor of ten increase or decrease of the parameters (a) $k_{a}$, (b) $k_{d}$, (c) $k_{t r}$, (d) $k_{t l}$, (e) $k_{\text {tll }}$ and (f) $\alpha$ from their best-fit values (Table 4) influences the predictions of the proposed ATRP model described in Section 3 of Supporting Information. The measured data points are same as in Figures 3 and 4 in the main text. Here, the solid, dashed and dashed-broken curves represent the ligand-to-copper ratios of $[\text { PMDETA }]_{\mathrm{o}}:[\mathrm{Cu}(\mathrm{I}) \mathrm{Br}]_{\mathrm{o}}=1: 1,2: 1$ and $4: 1$, respectively. $\mathrm{x}$ denotes the monomer conversion.

Section 6: Predictions of the proposed ATRP model (Section 3 of Supporting Information) for the number-average and weight-average chain lengths $\left(x_{n}\right.$ and $x_{w}$, respectively) and polydispersity index (PDI) for the PEO-PnBA product as functions of the monomer conversion $(x)$. 


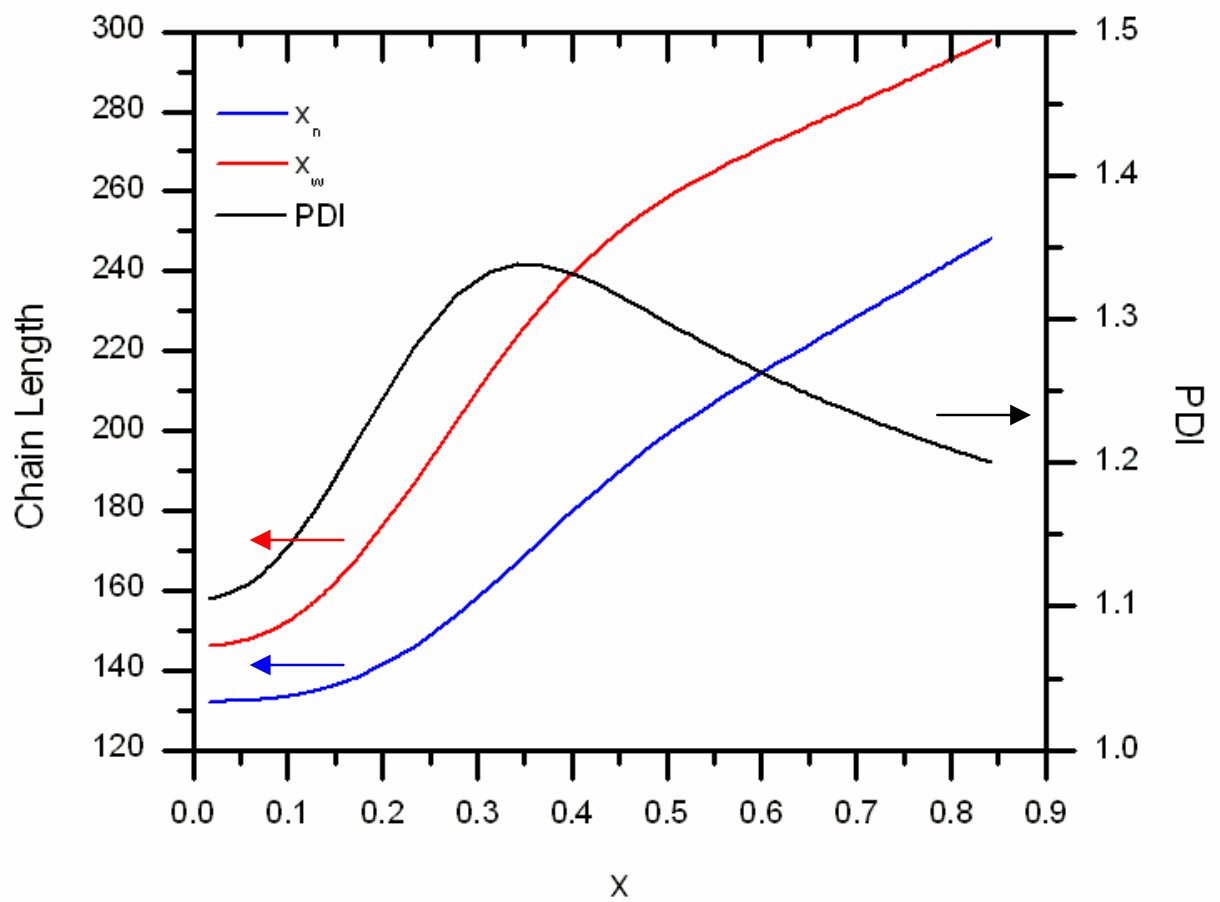

(a)

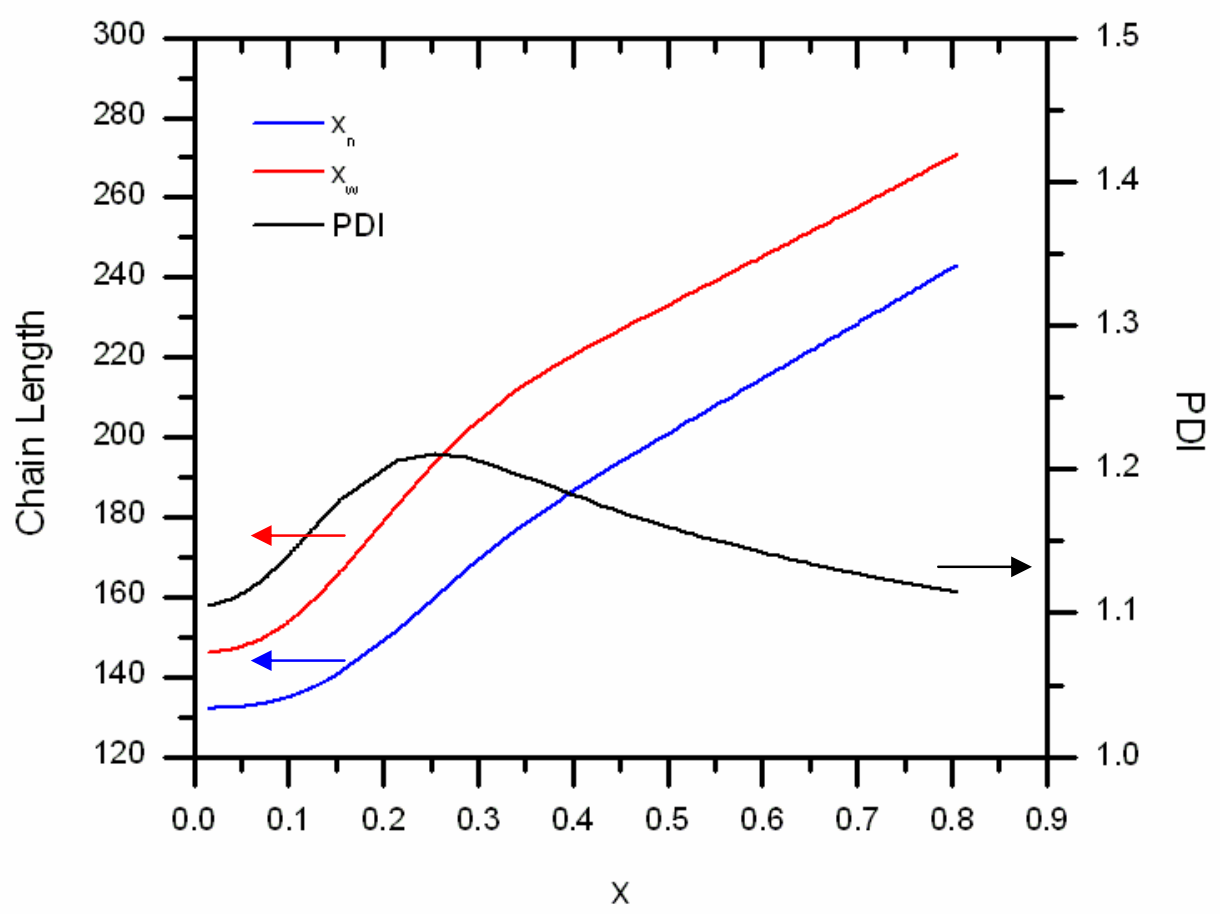

(b) 


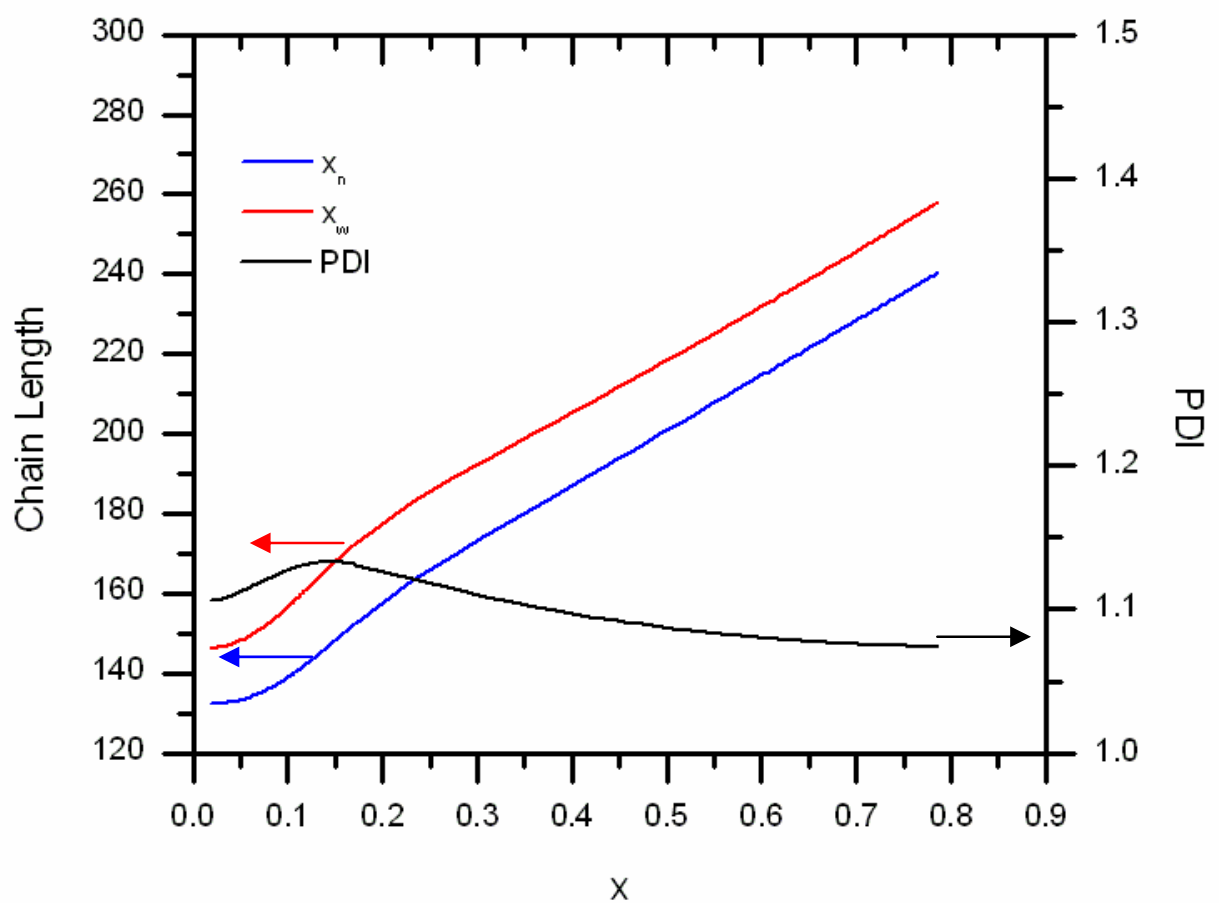

(c)

Figure S2. Evolution of cumulative chain lengths (i.e., $\mathrm{x}_{\mathrm{n}}$ and $\mathrm{x}_{\mathrm{w}}$ ) and polydispersity (PDI) for the ATRP of nBA using the PEO macroinitiator. Here, model predictions are shown for the three different ligand-to-catalyst ratios of $[\mathrm{PMDETA}]_{\mathrm{o}}:[\mathrm{Cu}(\mathrm{I}) \mathrm{Br}]_{\mathrm{o}}=$ (a) $1: 1$, (b) 2:1, and (c) 4:1. 